\title{
Preliminary Results of Terabit-per-second Long-Range Free-Space Optical Transmission Experiment THRUST
}

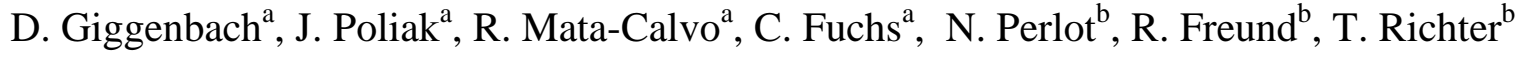 \\ ${ }^{\mathrm{a}}$ German Aerospace Center (DLR), Inst. of Comm. and Navigation, Oberpfaffenhofen, Germany \\ ${ }^{\mathrm{b}}$ Fraunhofer - Heinrich Hertz Institute, Berlin, Germany
}

\begin{abstract}
Future Very High Throughput Satellite Systems (VHTS) will perform at several Tbit/s throughput and thus face the challenge of limited feeder-link spectrum. Whereas with conventional RF feeder links several tens of ground gateway stations would be required, the total capacity can alternatively be linked through a single optical ground station using Dense Wavelength Division Multiplexing (DWDM) techniques as known from terrestrial fiber communications. While intermittent link blockage by clouds can be compensated by ground station diversity, the optical uplink signal is directly affected by scintillation and beam wander induced by the atmospheric index-of-refraction turbulence. The transmission system must be capable to mitigate these distortions by according high-speed tracking and fading compensation techniques. We report on the design of a near-ground long-range $(10 \mathrm{~km})$ atmospheric transmission test-bed which is, with its relatively low elevation of 1.8 degrees, exemplary for a worst case GEO uplink scenario. The transmitting side of the test-bed consists of a single telescope with a a fine pointing assembly in order to track the atmospheric angle-ofarrival and precisely aim towards the beacon of the receiver. On the other side of the test-bed, the receiver telescope is also capable of fine pointing by tracking the transmitted signal. The GEO uplink scenario is modelled by a precise scaling of the beam divergence and the receiver's field of view as well as by the beacon offset to model the point-ahead angle. In order to make the experimental test-bed correspond to an actual feeder link scenario, the link budget as well as the turbulence profile of the experimental scenario are modelled and compared to the GEO uplink. Several DWDM channels are multiplexed to reach the total link capacity of above one Tbit/s.
\end{abstract}

We report on the preparatory lab tests, and the free-space test-bed design and verification, of the Terabit Throughput Satellite Technology Project THRUST.

Keywords: Optical satellite feeder link, atmospheric beam distortions, beam pointing and tracking, multi-channel FSO by DWDM

\section{INTRODUCTION}

In Europe we currently see strong efforts to provide fast internet service to as many households as financially viable. Terrestrial cable and wireless techniques are being exploited to their physical maximum. Still we will also in long-term future see white spots on the connectivity map, especially in Eastern and Southern Europe. This situation applies even stronger for other densely populated areas on the globe like South America and Africa, which will see a few percent of the population being left without high-speed internet in the long term. Satellite communications from geostationary satellites (GEOs) can provide the required connectivity since it is not obstructed by any topological issues on ground. However currently existing communication satellite transmission technology cannot provide the required high throughput (which will easily go beyond $1 \mathrm{Tbit} / \mathrm{s}$ per satellite) to serve such large amounts of the population. New technological approaches are necessary, known as Very High Throughput Satellite System (VHTS), together with optimized data transfer protocols like DVB-Sx-RTS, plus the user-link cells on ground must be optimized for maximum frequency re-use [1][2][3]. For these systems, the data feeder link trunkline from the ground-hub to the GEO satellite becomes a major issue as illustrated in Figure 1: When sending the data in the radio frequency (RF) spectrum, each ground gateway is limited by its available spectrum, which is typically 3 to $5 \mathrm{GHz}$ only. Thus, several ground gateways 
are required to deliver the system throughput. This number reaches above 50 gateways already for 1 Tbit/s, plus additional backup stations will be required to compensate for local strong rain fading events.

When instead free-space optical communications technology (FSO) is used, more than one Tbit/s can be sent via only one Optical Ground Station (OGS) towards the GEO, this technology is called Optical GEO Feeder Link (OGEOFL). One however needs to compensate link blockage by clouds through large-scale OGS-diversity. By analysis of long-term cloud data it can be shown that for OGSs in the Mediterranean (or similar favorable areas between $20^{\circ}$ and $40^{\circ}$ latitude in the northern or southern hemisphere), around 11 gateway stations can be sufficient to fulfill availability requirements for satellite telecom systems, while this number can even be reduced when an inter-hemispherical ground network exploitats the anti-correlated seasonal weather statistics [4], [5], [6]. In general, above a throughput of roughly 300 Gbit/s, OGEOFLs require less ground stations than RF technology.

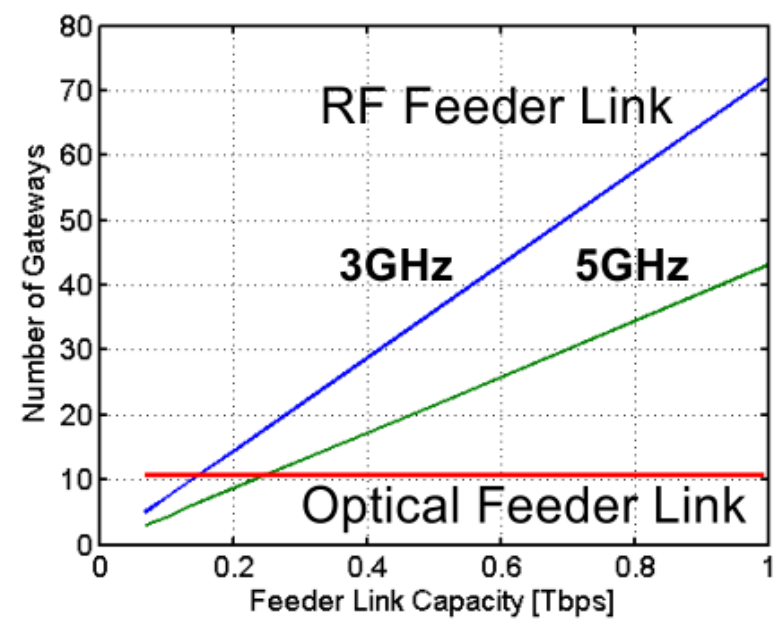

Figure 1. Number of gateways for RF feeder link technology versus optical feeder links (where number further reduces when an inter-continental OGS-network with hubs on northern and southern hemisphere can be established) [5]

Transmission technology for OGEOFLs will be employing components from terrestrial fiber communications in the DWDM-grid (Dense Wavelength Division Multiplexing), namely laser sources, optical fiber amplifiers, and optical multiplexers and demultiplexers. The clear-sky attenuation of the atmosphere features a minimum exactly around the DWDM spectral region from 1530nm up to 1680nm (Figure 2).

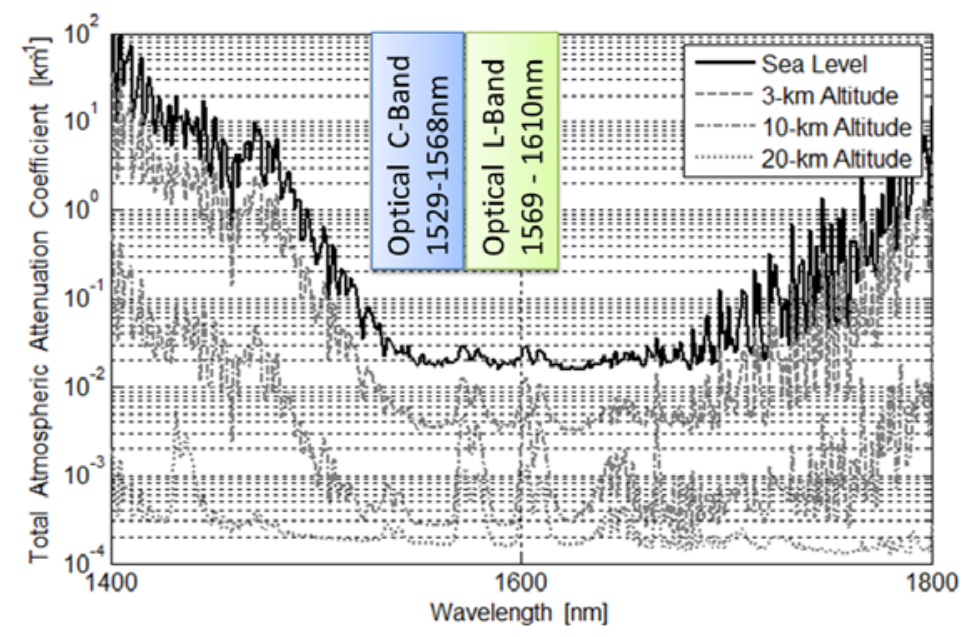

Figure 2. Atmospheric attenuation coefficient in the area of DWDM wavelengths for different altitudes 
One major challenge however to be solved for OGEOFL is the clear-sky turbulence of the free atmosphere, caused by index-of-refraction turbulence cells (IRT) [7]. These cells cause intensity fluctuations and wavefront distortions of the propagated laser signal. While the IRT effect in the downlink from the GEO to the OGS can be compensated by means of aperture averaging and adaptive-optics wavefront correction, the uplink beam suffers from stochastic direction deviations called beam-wander, which are hard to compensate with standard techniques. This beam wander-effect thus can cause a major degradation of the overall link budget.

Some demonstrations have been performed to show the feasibility of high-throughput DWDM transmission through the atmosphere, however these have been limited to short ranges where the atmospheric IRT is not yet a limiting factor for system performance [8][9]. With the THRUST-testbed we will target specifically the effect of IRT onto the Tbit/sDWDM signal beam in the uplink.

\section{OPTICAL GEO FEEDER LINK TESTBED DESCRIPTION}

\section{Tbit/s optical data transmission system applied to free-space optical feeder links:}

The atmospheric THRUST-Testbed features a similar communication system as the target scenario of future groundGEO optical feeder links: The intended setup for such a Tbit/s system is schematically depicted in Figure 3. Forty optical signals each carrying an ON/OFF modulated 43-Gbps data channel are multiplexed into one single mode fiber using DWDM technology. An erbium-doped fiber amplifier (EDFA) amplifies these signals and the opto-mechanical assembly (OMA) directs the beam towards the receiving terminal. There the received fraction of the signal is steered into a singlemode fiber by the receiver-OMA. This signal is amplified to gain sensitivity and to equalize atmospheric power fades. Finally, a DWDM de-multiplexer separates the different channel wavelengths which are detected by individual directdetection receiver frontends.
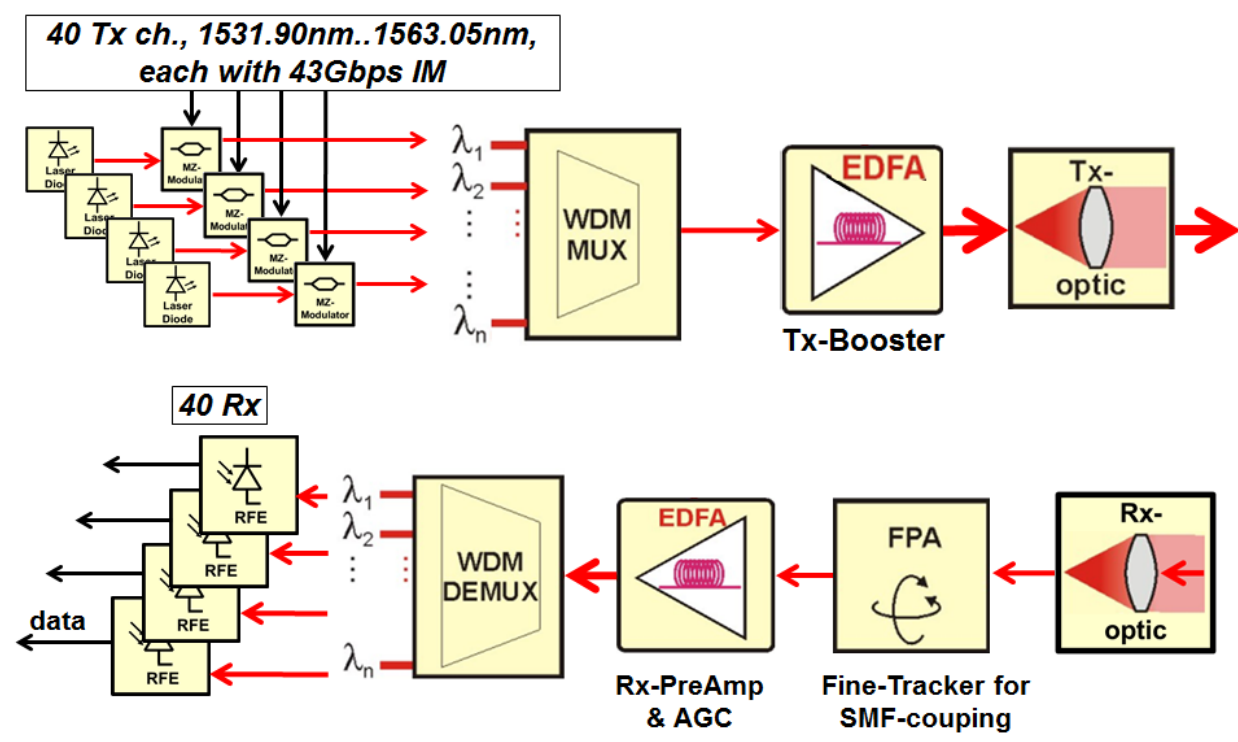

Figure 3. The DWDM signal chain of the THRUST testbed resembles the future target scenario of optical GEO feeder links. Only one link direction is shown.

The OGEOFL Testbed scenario and design were first introduced in [10]. There, a possible technical realization of the testbed was introduced and a scenario analysis with emphasis on the $\mathrm{C}_{\mathrm{n}}{ }^{2}$-profile of the testbed was performed and compared with the actual OGEOFL scenario. As a further means to reduce uplink fading, transmitter diversity schemes need to be considered to be tested in a second stage with the testbed [11]. 


\section{Testbed Transmitter Section Description:}

The transmitter part of the OMA, also referred to as an optical ground station (OGS), is shown in the left part of Figure 4. All amplified modulated DWDM optical signals are connected to the OMA using a single-mode fiber (SMF). The output light is collimated and finely steered to the precise direction using fine-steering tip-tilt mirror (FSM). The signal is then precisely aligned at the input of the refractor telescope of the OGS. The OGS telescope consists of a set of diffraction-limited lenses which produce a Gaussian transmitted beam at the output with a divergence in the order of tens of microradians.

The OGS telescope at the same time receives the incoming beacon from the receiver, which has a wavelength of $1310 \mathrm{~nm}$. The beacon beam can be, due to its divergence of several milliradians, modelled as a spherical wave. The beacon is offset from the receiver by a distance, which corresponds to the point-ahead angle of $18 \mu \mathrm{rad}$, simulating the actual OGEOFL scenario. The incoming beacon beam is then reflected by the FSM to a dichroic mirror, which reflects the beacon beam towards a position-sensitive device (PSD), providing a reference to the pointing control loop. In the initial phase of acquisition as well as during larger misalignments, the whole OMA is steered by a coarse-pointing assembly (CPA).

\section{Receiver Section}

The receiver part of the OMA, also referred to as a satellite unit (SAT), is shown in the right part of Figure 4. Similarly to the OGS, in the SAT the received optical signal is coupled using a refractive Keplerian telescope and aligned to FSM. The beam is then reflected to a bulk optical coupler, where one part is detected at a PSD and serves as pointing reference. The other part of the beam is then coupled into a SMF.

The coupling into the SMF is done by means of coupling optics, which is defined by the numerical aperture $N A_{\mathrm{t}}$. It is important to match the $N A_{\mathrm{t}}$ and the numerical aperture of the fiber $N A_{\mathrm{f}}$.

The coupled optical signal is then guided to a 10/90 fiber splitter, where $10 \%$ of the optical power is observed and provides feedback for the optimization of the pointing (closed-loop). The measured power dynamics also carries information about the instant state of the optical link and of the atmospheric conditions. The remaining part of the optical power is then guided to the communications system, where its optical spectrum is observed before it is pre-amplified and analyzed for the presence of errors.

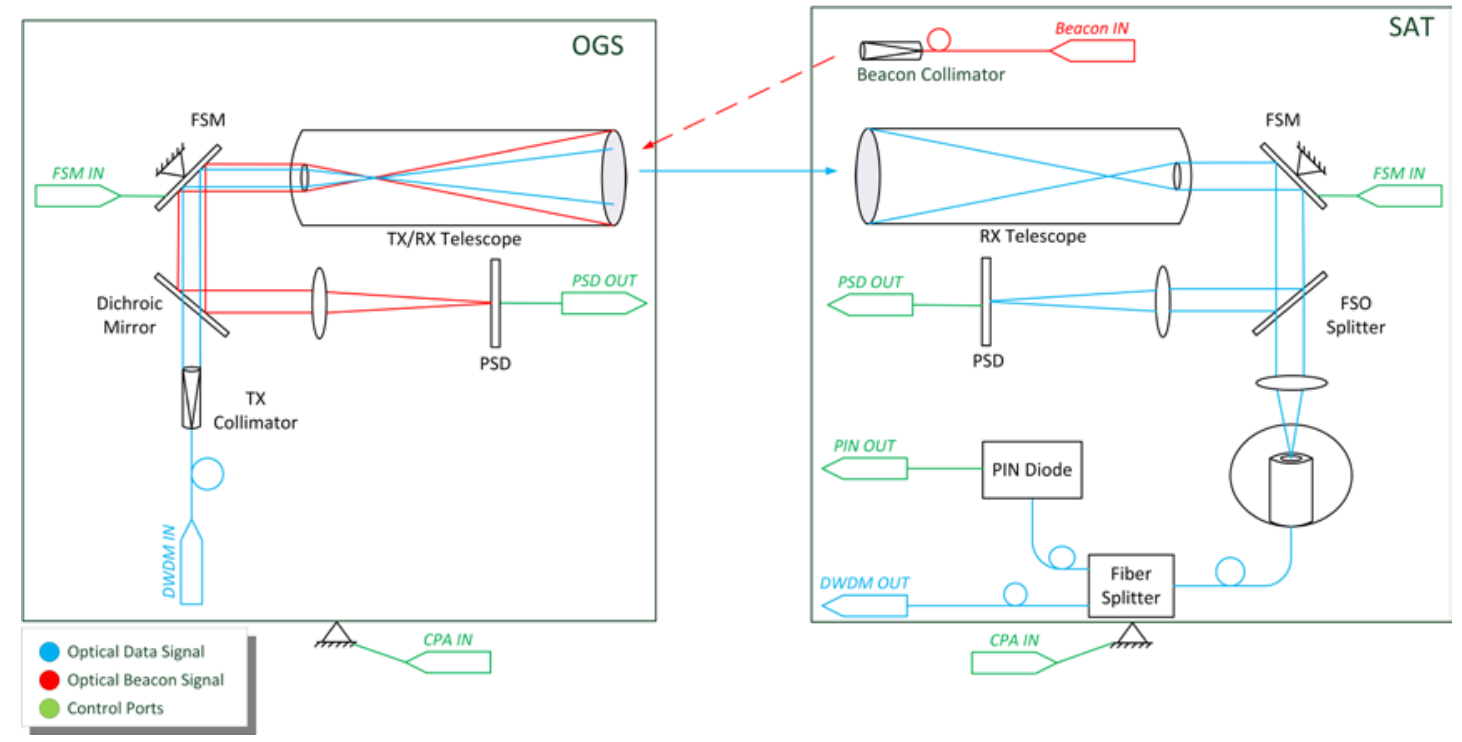

Figure 4. THRUST testbed hardware setup block diagram 

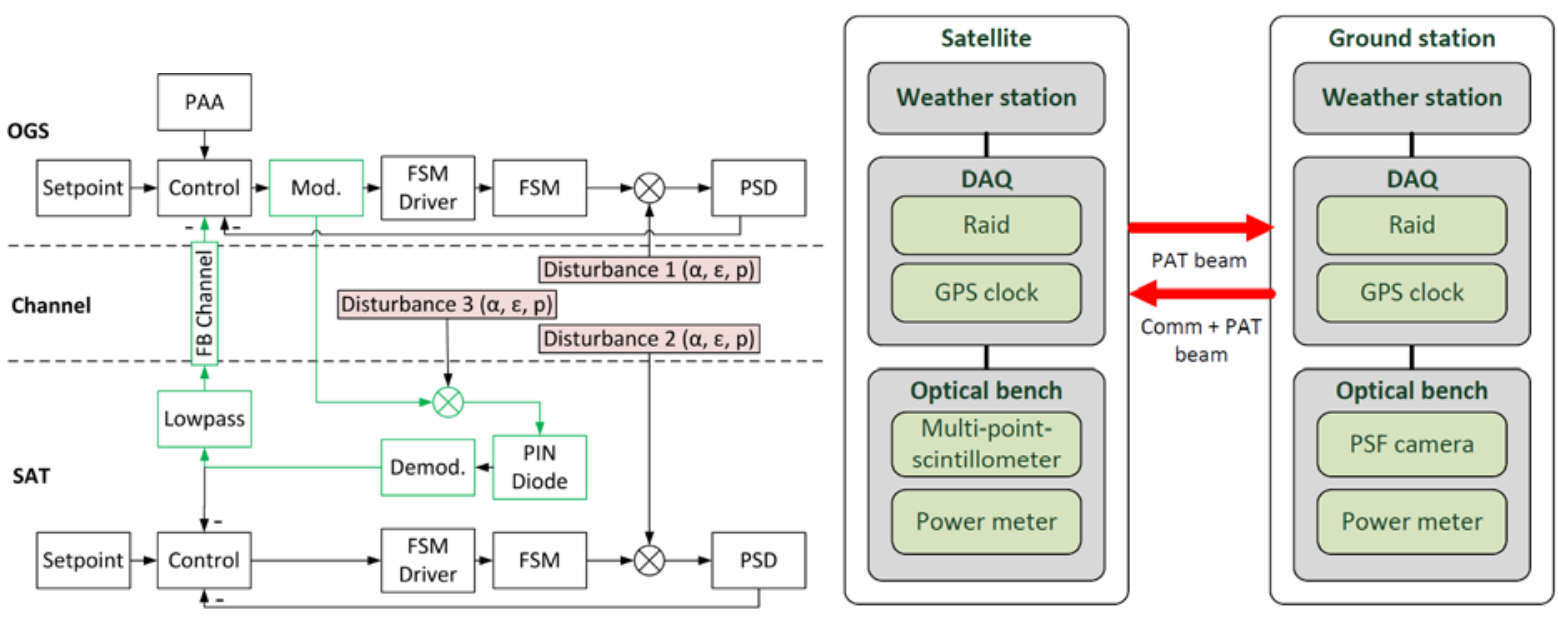

Figure 5. System control and sensor block diagram

Figure 5 shows the concept of the control of the OMA in the testbed. The output of the PSD on both OGS and SAT terminals provide feedback to the control systems to steer the FSM to reflect the beam to a defined setpoint. Main disturbances are results of power scintillation $p$ as well as of the deviation in azimuth $\alpha$ and elevation $\varepsilon$. In order to achieve the expected pointing performance, the beacon offset, corresponding to the point-ahead angle (PAA), needs to be compensated for.

The system also includes closed-loop pointing functionality by means of received optical power in the SAT and steering the OMA towards OGS to improve the overall pointing performance. The small dither signal is introduced to the steering in the Modulator (Mod.), cf. Figure 5. Its effect is then observed in the demodulated output signal and feedback can be provided to the OGS by means of an additional link or by means of modulating the information on the beacon beam. The latter is not considered due to its possible influence on the metrology system results.

In order to fully verify the performance of the optical data transmission and of the OMA, a complete understanding of the atmospheric and measurement conditions is necessary. For that purpose, an atmospheric metrology system has been designed. At the OGS, a focal camera (or PSF camera) is employed to observe the incoming beacon beam in the focus of a telescope. From the observation of focal speckles, one can extract information about the instant value of the Fried parameter in the downlink part of the link.

In the other direction (uplink), the information about the Fried parameter is not possible to measure due to the link geometry. However, necessary information about beam wander, beam spread and scintillation can be extracted from a so-called multi-point scintillometer.

At the same time, information about the coupled optical power as well as the weather condition will be monitored and stored for post-processing. One can also capture the signals corresponding to the steering of the CPA and the FSM as well as to the beam position on the PSD. Due to the distance between the OGS and the SAT, one has to also find alternative means of synchronization of the measurements at both sites. Direct connection is not always applicable; therefore precise absolute timestamp will be obtained from the GPS signal and attached to the measured data.

\section{Linkbudgets}

A comparison of the THRUST-testbed link budget with the link budget of the target application of an optical GEO feeder link in Table 1 indicates similarities and differences, which are mainly in the transmit power, beam divergence, and receiver aperture. A line rate of $43 \mathrm{Gbit} / \mathrm{s}$ is assumed before FEC, to allow for a channel rate of $40 \mathrm{Gbit} / \mathrm{s}$ after application of FEC. 
Table 1. Link budget analysis for 40 DWDM uplink channels at 40Gbit/s each (43Gbit/s line rate)

\begin{tabular}{|c|c|c|}
\hline Parameters & THRUST-Testbed & OGEOFL \\
\hline Number of channels [-] & \multicolumn{2}{|c|}{40} \\
\hline Mean optical power per channel [dBm] & 17 & 47 \\
\hline Wavelength [nm] & \multicolumn{2}{|c|}{ 1532..1563 } \\
\hline Transmitted beam divergence [ $\mu \mathrm{rad}]$ & 50 & 5 \\
\hline Line Rate before FEC [Gbit/s] & \multicolumn{2}{|c|}{43} \\
\hline OGS-transmittance [-] & \multicolumn{2}{|c|}{$80 \%$} \\
\hline Average mispointing \& tracking loss at OGS [-] & $50 \%$ & $70 \%$ \\
\hline Link distance $[\mathrm{km}]$ & 10.5 & 36000 \\
\hline Receiver telescope diameter [m] & 0.125 & 0.400 \\
\hline SAT-transmittance [-] & \multicolumn{2}{|c|}{$80 \%$} \\
\hline Tracking and coupling efficiency at SAT [-] & $40 \%$ & $70 \%$ \\
\hline Atmospheric attenuation loss [dB] & \multicolumn{2}{|c|}{1.3} \\
\hline Scintillation loss $[\mathrm{dB}]$ & \multicolumn{2}{|c|}{6} \\
\hline Mean sensitivity [dBm] @ BER, before FEC [-] & \multicolumn{2}{|c|}{$-27 @ 10^{-3}$} \\
\hline Estimated Link Margin [dB] & 13.4 & 6.6 \\
\hline
\end{tabular}

\section{Emulation of the turbulence effects: scintillation and pointing-errors}

The target scenario refers to a real implementation of Tbit/s-feeder link from an optical ground station to a laser terminal embarked on a geostationary satellite, as well as in the opposite direction. A major difference between the satellite link and the horizontal link is the PAA between the incoming signal from the satellite and the outgoing from the ground station towards the satellite. This angle is required to compensate for the movement of the satellite $(\sim 7000 \mathrm{~km} / \mathrm{h})$ during the time-of-flight of the signals. While this angle is known to be $\sim 18 \mu \mathrm{rad}$ (exact value depending on link elevation and latitude of the OGS), it causes a dynamic discrepancy of the incoming angle-of-arrival $\alpha_{\text {AoA }}$ due to atmospheric turbulence, and the atmospheric beam wander angle $\alpha_{B W}$ of the outgoing beam. This will prevent perfect pointing of the outgoing beam and thus put a limitation to the link budget. This effect can however be emulated to a certain extend by offsetting, at the space terminal, the beacon aperture from the receive aperture.

In applying pointing-by-tracking, the satellite uplink-pointing uses the tracked downlink signal to pre-compensate the beam-wandering induced by the atmosphere. Pointing-by-tracking is then possible when the PAA is within the cone of the tilt-isoplanatic angle (TIA), i.e. the cone where the turbulence induced tilt remains correlated. In Figure 6, the main involved parameters in pointing-by-tracking are represented: the point-ahead angle $\alpha_{\text {PAA }}$, the tilt-isoplanatic angle $\alpha_{\text {TIA }}$, the full-width half-maximum uplink beam divergence $\theta_{\text {FWнM }}$, the downlink angle-of-arrival $\alpha_{A \circ A}$, the uplink-tilt or beam wander $\alpha_{B W}$ and the isoplanatic angle (IPA). 


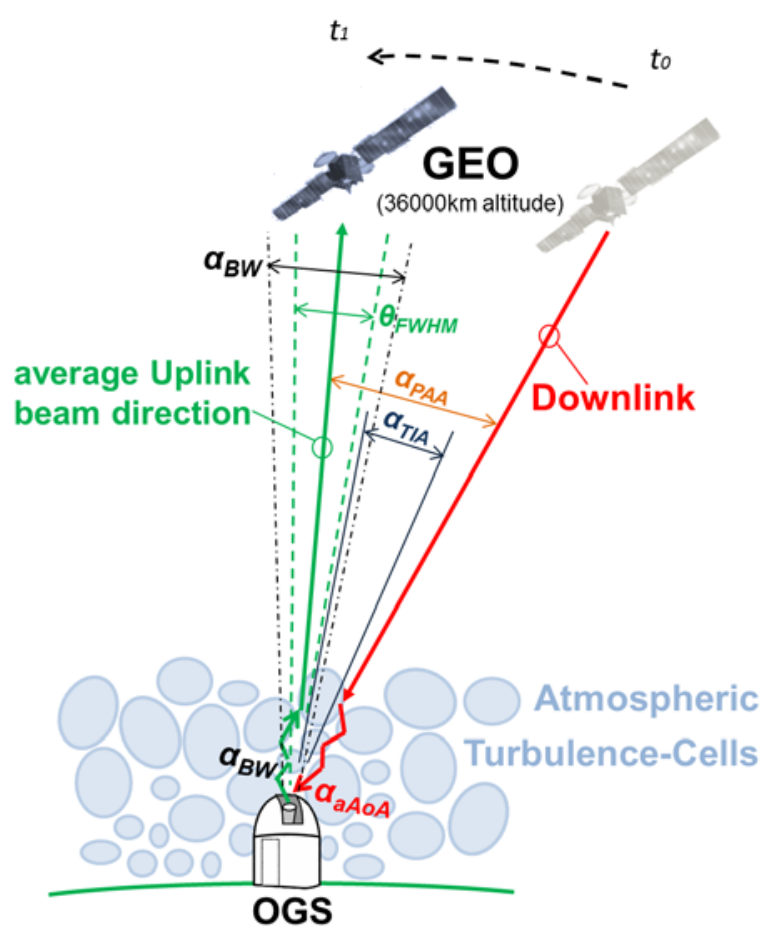

Figure 6. The point-ahead angle is typically larger than the atmosphere's tilt-isoplanatic angle, causing reduced quality of pointing-by-tracking. This is simulated in the testbed by an off-axis beacon source with variable off-set.

Two effects need to be emulated in the horizontal link test-bed to get a realistic received signal: scintillation and pointing errors. Scintillation is the result of a self-interference process due to phase distortions produced by small-scale index-ofrefraction fluctuations. Usually, a lognormal distribution of the intensity fluctuations is assumed for satellite link elevations above $30^{\circ}$ and pre-distortion adaptive optics or transmitter diversity techniques are needed to minimize the uplink scintillation. The satellite aperture of a GEO satellite can be represented as a point receiver, i.e. the receiver diameter is much smaller than the intensity speckles of the uplink beam. This can be modelled using a telescope aperture smaller than the intensity correlation radius, to avoid any aperture averaging. Pointing errors, due to beam-wandering, cause strong-fading of the received signal. Although pointing-by-tracking is used, to minimize beam-wandering, some pointing error remains because the correlation decreases due to the PAA. A beta-distribution is assumed for modelling the intensity fluctuations produced by the remaining pointing error. Since the horizontal link does not require any PAA, this condition can be simulated by separating the downlink beacon signal by $18 \mathrm{~cm}$ (18 $\mu \mathrm{rad}$ after 10-km link distance) and applying $18 \mu \mathrm{rad}$ offset to the uplink signal.

\section{UPLINK AND DOWNLINK IRT ASSESSMENT RESULTS}

The scintillation strength in the testbed topology has been assessed by a simulation of the scenario and by power variation measurements. Figure 7 shows the height profile from the DLR site in Weilheim-Lichtenau to Hohenpeißenberg. The altitude of the terrain is increasing from around $600 \mathrm{~m}$ at Lichtenau to about $900 \mathrm{~m}$ on the mountain top station. The height of the line-of-sight (LOS) above ground in the middle part of the link varies from $50 \mathrm{~m}$ to $150 \mathrm{~m}$. 


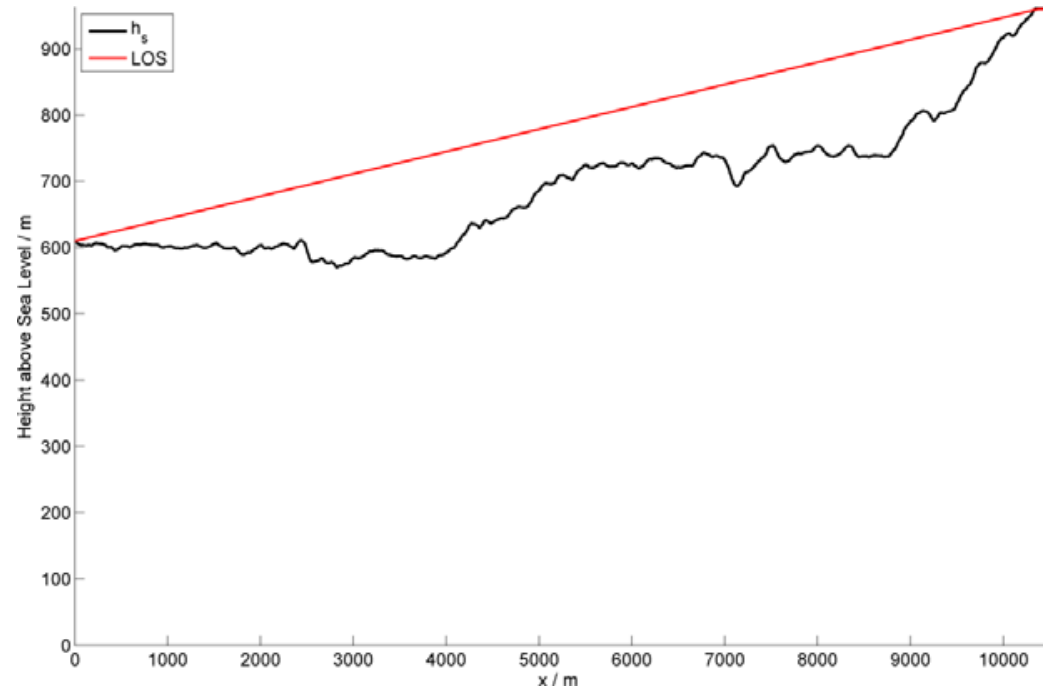

Figure 7. Height profile for ground-to-ground link from DLR-Weilheim to DWD’s site at Hohenpeißenberg

The turbulence profile has been estimated based on a modified Hufnagel-Valley model as described in [12]. It was calculated according to the path-height along the link. Furthermore, it was compared to several ground-GEO scenarios for both a best- and worst-case. The chosen parameters of the turbulence profile are stated in Table 2.

Figure 8 shows the calculated turbulence profiles along the LOS (z) for the ground-ground scenario (dotted black and blue lines) and compares them to different ground-GEO scenarios at $2^{\circ}, 10^{\circ}$ and $30^{\circ}$ of elevation. It can be seen that the gradient of the ground-ground turbulence profile behaves very similar to a ground-GEO scenario at $2^{\circ}$ of elevation. The gradient of ground-GEO-turbulence profiles at $10^{\circ}$ or even $30^{\circ}$ of elevation, as is most relevant for optical GEO feeder links, is much steeper. This indicates that the ground-ground testbed will represent a worst-case turbulence gradient.

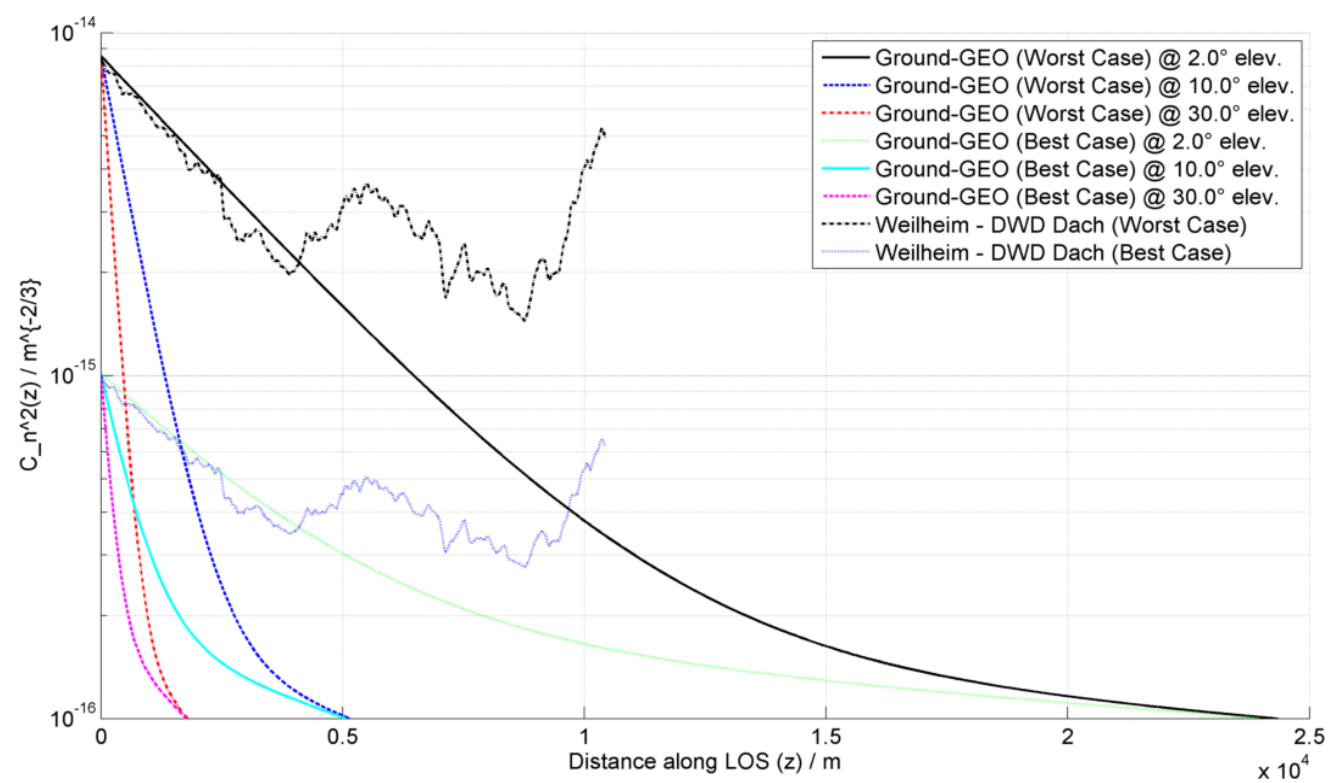

Figure 8. Turbulence profiles along the line-of-sight (LOS) for the ground-ground scenario, compared to ground-to-GEO scenarios with $2^{\circ}, 10^{\circ}$ and $30^{\circ}$ of elevation, compare Table 2. 


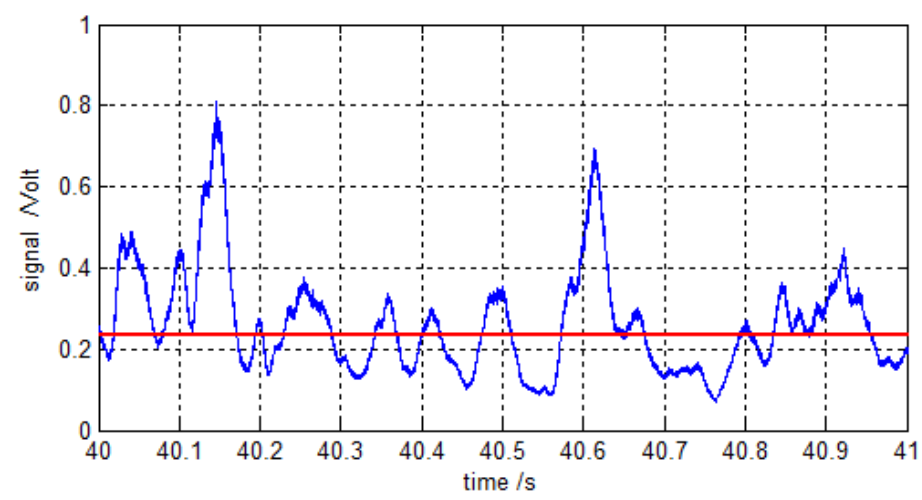

Figure 9. Typical 1-s trace of uplink power fading vector in the THRUST-Testbed, at summer daytime with PSI=0.30. Red line indicates mean value.

Preliminary tests were carried out to assess the turbulence strength in the uplink from the OGS simulator at Lichtenau to

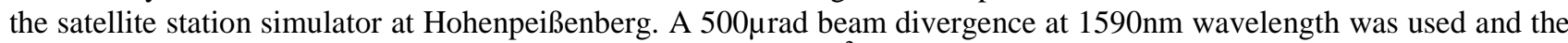
tests were carried out at daytime in summer, indicating a high- $\mathrm{C}_{\mathrm{n}}{ }^{2}$ IRT situation.

Table 2. Turbulence profile parameters, analytical values [7], and measured values. Autocovariance-width (ACOV) of the power vector serves as measure for fading time. (PSI: power scintillation index, UL: uplink, DL: downlink)

\begin{tabular}{|c|c|c|c|c|}
\hline & Unit & Best case profile & Worst case profile & Measured in testbed \\
\hline Ground value A & {$\left[\mathrm{m}^{\wedge}-(2 / 3)\right]$} & 2e-15 & $2 e-14$ & - \\
\hline Pseudo wind speed v & {$[\mathrm{m} / \mathrm{s}]$} & 21 & 21 & - \\
\hline Rytov-index UL, testbed & 1 & 0.774 & 5.54 & - \\
\hline Rytov-index DL, testbed & 1 & 0.607 & 4.04 & - \\
\hline Rytov-index, GEO $20^{\circ}$ el. & 1 & 0.35 & 0.35 & - \\
\hline PSI $(5 \mathrm{~cm})$ UL, testbed & 1 & 0.266 & 0.958 & {$[0.20 \ldots 0.30] ;[0.27 \ldots 0.46]$} \\
\hline PSI (5cm) DL, testbed & 1 & 0.211 & 0.878 & {$[0.12 \ldots 0.20]$} \\
\hline PSI $(5 \mathrm{~cm}) \mathrm{DL}, \mathrm{GEO} 20^{\circ} \mathrm{el}$. & 1 & 0.34 & 0.36 & - \\
\hline$r_{0}$ UL, testbed & $\mathrm{cm}$ & 24 & 7.7 & - \\
\hline$r_{0}$ DL, testbed & $\mathrm{cm}$ & 12 & 3.8 & - \\
\hline $\mathrm{r}_{0}$ DL, GEO $20^{\circ} \mathrm{el}$. & $\mathrm{cm}$ & 26 & 14 & - \\
\hline ACOV-FWHM UL, testbed & $\mathrm{ms}$ & - & - & 6 (wind) ... 35 (calm) \\
\hline ACOV-FWHM DL, testbed & $\mathrm{ms}$ & - & - & 7 (wind) ... 20 (calm) \\
\hline $\begin{array}{c}\text { mean 3dB fades, UL } \\
\text { GEO } 20^{\circ} \text { el. }\end{array}$ & $\mathrm{ms}$ & - & - & 9 , from [12] \\
\hline
\end{tabular}


The measurements do not yet reflect the effect of uplink beam wander with tracked or untracked transmitter direction, these will be assessed with the final system which also provides a pointer-tracker opto-mechanics.

\section{EXPERIMENTAL PERFORMANCE ANALYSIS OF A PREAMPLIFIED DIRECT- DETECTION RECEIVER UNDER EMULATED FADING}

In this section we report on the impact of power fluctuations on the reception of a 43-Gb/s non-return-to-zero (NRZ) OOK signal by a pre-amplified direct-detection receiver. The emulated fluctuating received power is representative of the turbulent channel and its impact is evaluated in terms of BER statistics.

The experimental setup is depicted in Figure 9. At the transmitter, the output of a continuous-wave laser is intensity modulated in a Mach-Zehnder modulator (MZM). The MZM is driven by a bit-pattern generator (BPG) which provides a pseudo random binary sequence of length $2^{31}-1$. In the channel stage, power fluctuations are applied to the signal by a fading emulator, based on a voltage-controlled, fast $(<1-\mu$ s response time), SMF coupled variable optical attenuator, which emulates IRT-induced intensity fluctuations. The generated power fluctuations have a correlation time of a few milliseconds [12] and two different scintillation indices ( 0.27 and 1.8 , best and worst case) were considered. Furthermore, the mean received optical power $\left(P_{\text {rec }}\right)$ in front of the receiver is adjusted by use of a variable optical attenuator (VOA). In the receiver, the signal is amplified to about $12 \mathrm{dBm}$ by a cascade of two EDFAs. The EDFAs are operated in a constant output power mode. An optical band-pass filter is used between the two EDFAs to suppress outof-band amplified spontaneous emission noise from the first EDFA. The signal is split by a 3-dB coupler, and detected on two photodetectors (50-GHz bandwidth). One of the two photodetectors is connected to the error analyzer (EA) for BER measurements. The clock signal for the EA is provided by the second photodetector and an electrical 43-Gbps clock-recovery (CR) with a locking time of $\sim 100 \mu$ s.

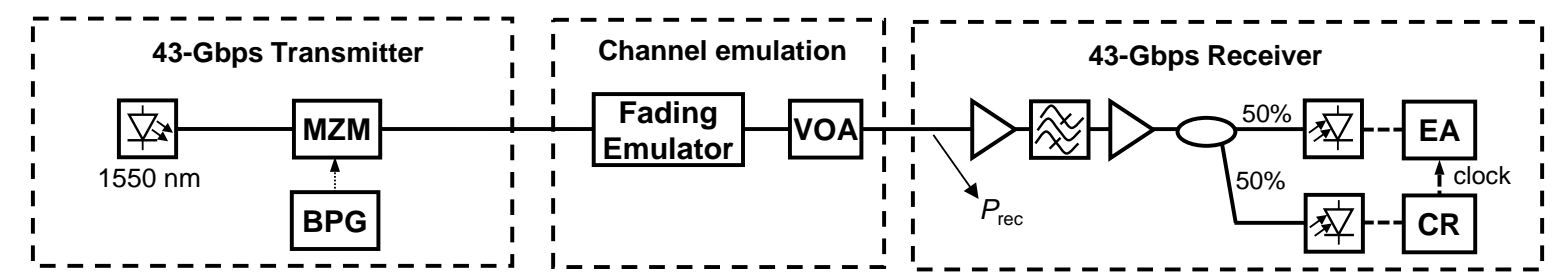

Figure 10. Experimental setup

First, we measured the BER performance of the system without fading (i.e. fading emulator inactive) which is shown in Figure 11 (a). In this case, a received power of $-24 \mathrm{dBm}$ is required for a BER of $10^{-9}$, while $-35.7 \mathrm{dBm}$ is sufficient for a BER of $10^{-3}$.

Second, we used the fading emulator to generate power fluctuations. For the two considered scintillation indices, 0.28 and 1.8, the EDFAs in the receiver maintained an almost constant output power on the photodetectors with a measured mean-normalized variance of 0.02. Along with the fluctuating power at the receiver input, the BER variations essentially followed the static BER performance curve. We measured the BER every $500 \mathrm{~ms}$ over a time of about 30 min. The gating time of the error analyzer was set to $10 \mathrm{~ms}$ which allowed us to evaluate BERs down to $2 \times 10^{-9}$ (with 1 error per $10 \mathrm{~ms}$ ) and up to $10^{-2}$ (due to the limited memory depth of the EA for error counting). In total, more than 3000 sequential BER measurements were used to derive BER statistics. The results are summarized in Figure 11 (b) and (c). The average received power was set to $-27 \mathrm{dBm}$. The distribution of the BER is determined by the distribution of the power fluctuation at the fading emulator. In case of the weak power fluctuation, we observe BERs $<10^{-3}$ for less than $1 \%$ of the measurements. However, in case of the strong power fluctuation, this value increased to $>30 \%$. We observed a large number of measurements in which the BER is below $10^{-2}$. Such cases result from insufficient OSNR at low received powers. We observed also cases where the clock recovery failed to lock. These CR unlocking events are supposedly related to rare cases in which the input signal to the CR is too low because the EDFAs have failed to compensate for the 
power fades as well as limited OSNR. In such cases a relocking of the clock-recovery for about $100 \mu$ s is required. Nonetheless, we observed stable locking of the clock recovery down to a power of $-44 \mathrm{dBm}$ at the receiver input.

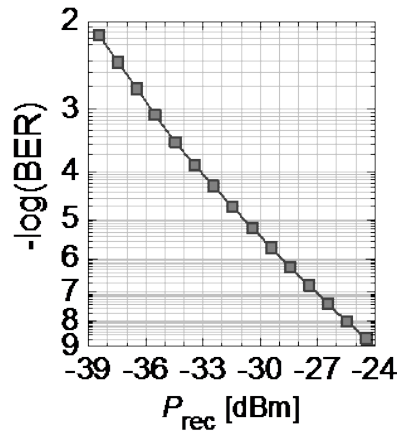

(a)

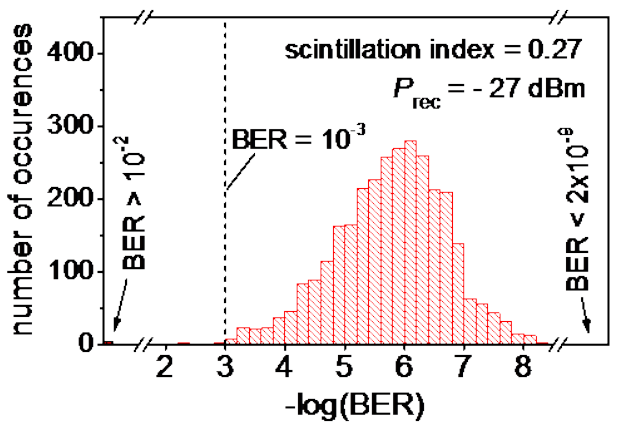

(b)

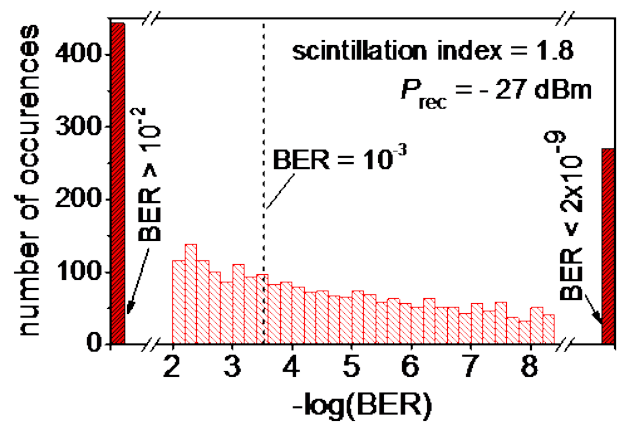

(c)

Figure 11. (a) BER performance without fading for different received powers (b) BER histogram under weak power fluctuations (scintillation index of 0.27). (c) BER histogram under strong power fluctuations (scintillation index of 1.80).

For both (b) and (c), the gating time of the error analyzer was set to $10 \mathrm{~ms}$, limiting the range of measurable BERs.

\section{OUTLOOK ON FURTHER LINK ASSESSMENT}

The knowledge of the uplink received signal behavior is of great interest in the design of the future optical communications terminals for the GEO satellite feeder links. The impact of the PAA on the pointing errors and on the signal statistics of the scintillation and the remaining beam wander needs to be completely characterized. The testbed presented in this paper allows investigating these turbulence effects and the techniques to minimize them.

This measurement setup has three main objectives: to demonstrate an optical communications link through the atmosphere with a throughput over 1 Tbit/s under a realistic turbulence environment, to characterize the communications link together with atmospheric turbulence and to develop mitigation techniques to minimize the turbulence impact in the received signal. The first step after the system validation which is currently ongoing is to demonstrate more than 1 Tbit/s communication link in October 2015, in the horizontal link Weilheim-Peißenberg. Another measurement campaign is planned then in the beginnings of 2016 to completely characterize the turbulence conditions during the communications link. The design of the mitigations techniques system is ongoing and field demonstrations are planned for June 2016. 


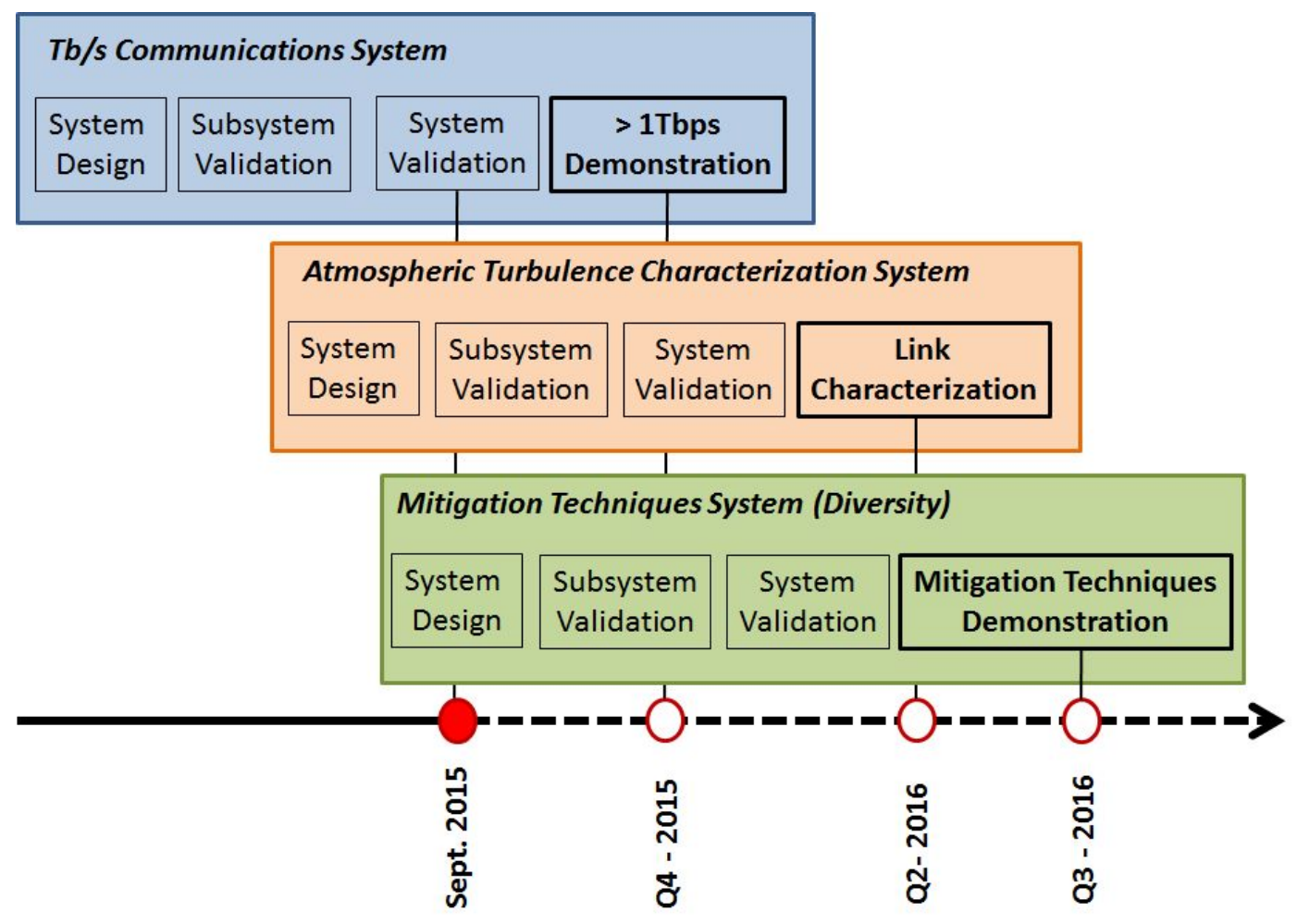

Figure 12. Timeline of THRUST-testbed experiment.

\section{ACKNOWLEDGEMENTS}

The kind support by the German Weather Service (Deutscher Wetterdienst, DWD) at the mountain-side station Hohenpeißenberg is deeply acknowledged. We also want to thank our colleagues from German Space Operations Center (GSOC) at DLR-site Weilheim for their support in installing the ground-site simulator station.

\section{REFERENCES}

[1] E. Lutz, "Towards the Terabit/s Satellite - Interference Issues in the User Link”, International Journal of Satellite Communications and Networking, 2015

[2] E. Lutz, “Achieving a terabit/s GEO satellite system. 19th Ka and Broadband Communications”, Navigation and Earth Observation Conference: Florence, Italy, Oct. 14-17, 2013; 509-516

[3] Broadband Access via Integrated Terrestrial and Satellite Systems (BATS), "ICT-2011.1.1 BATS D4.1: Satellite Network Mission Requirements” European Project, Tech. Rep., 2013

[4] ESA Artes projects UTMBeam and Terasat, at http://artes.esa.int/projects/

[5] D. Giggenbach, E. Lutz, J. Poliak, R. Mata-Calvo, C. Fuchs, “A High-Throughput Satellite System for Serving whole Europe with Fast Internet Service, Employing Optical Feeder Links” ITG-Conference "Breitbandversorgung in Deutschland”, April 20-21, Berlin, 2015

[6] D. Giggenbach, "Tutorial: Optical Free Space Links for Satellite-Ground Communications", 7th Advanced Satellite Multimedia Systems Conference, Livorno, Sept. 2014

[7] L. Andrews and R. Phillips, “Laser Beam Propagation through Random Media”, SPIE Press, 2005 
[8] E. Ciaramella, Y. Arimoto, G. Contestabile, M. Presi, A. D’Errico, V. Guarino, and M. Matsumoto, ”1.28 Terabit/s (32x40 Gbit/s) WDM Transmission System for Free Space Optical Communications”, IEEE Journal on selected areas in communciations, Vol. 27, NO. 9, December 2009

[9] Giorgia Parca, Ali Shahpari, Valeria Carrozzo, Giorgio Maria Tosi Beleffi, Antonio L. J. Teixeira, ”Optical wireless transmission at 1.6-Tbits $(16 \times 100 \mathrm{Gbit/s})$ for next-generation convergent urban Infrastructures”, Optical Engineering 52(11), November 2013

[10] J. Poliak, D. Giggenbach, F. Moll, F. Rein, C. Fuchs, R. Mata Calvo “Terabit-Throughput GEO Satellite Optical Feeder Link Testbed”, CONTEL, Graz 2015

[11]A. Mustafa, D. Giggenbach, J. Poliak, A. Shrestha, R. Mata-Calvo, C. Fuchs, "Lab implementation of 10Gbps/channel Optical Transmitter Diversity scheme for Geostationary Satellite Feeder Links”, 16th ITG Symposium on Photonic Networks, Leipzig, Germany, 2015

[12] R. Mata-Calvo, P. Becker, D. Giggenbach, F. Moll, M. Schwarzer, M. Hinz, Z. Sodnik, “Transmitter diversity verification on ARTEMIS geostationary satellite“, SPIE Photonics West, Feb. 2014

[13] S. Dimitrov, B. Matuz, G. Liva, R. Barrios, R. Mata-Calvo, D. Giggenbach, "Digital Modulation and Coding for Satellite Optical Feeder Links”, 7th Advanced Satellite Multimedia Systems Conference (ASMS), Livorno, Italy, Sept. 2014 